\title{
Metodología de Diseño de un Deshidratador Solar Indirecto de Frutos por Convección Forzada
}

\author{
José Cabrera Escobar, \\ Raúl Cabrera Escobar, \\ Darío Guamán Lozada, \\ Diego Cajamarca Carrazco, \\ Gustavo Carrera Oña,
} Escuela Superior Politécnica de Chimborazo, Riobamba, Ecuador

Doi: 10.19044/esj.2019.v15n18p140 URL:http://dx.doi.org/10.19044/esj.2019.v15n18p140

\section{Resumen}

La presente investigación pretende ser una guía para diseñar un deshidratador solar por convección forzada que sea capaz de funcionar sin energía de la red eléctrica, únicamente con energía solar, este diseño pretende ser la solución a los productores del campo. Para esta investigación se le dividió al deshidratador solar en 4 partes fundamentales que van a ser objeto de estudió las cuales son: colector solar, cámara de deshidratación, ventilador y panel fotovoltaico. Al finalizar la investigación se obtiene una guía práctica para el diseño de un deshidratador solar, tomando como referencia que Insolación Global promedio para Ecuador es de 4574,99 Wh/m2/día, si se requiere diseñar un deshidratador en otro país se debería consultar este dato para continuar con el diseño, también se llega a la conclusión que para tener un buen producto deshidratado no solo es necesario tener un deshidratador en excelentes condiciones si no también darle la adecuada preparación a nuestra materia prima.

Palabras clave: Panel Solar, deshidratador, cámara de deshidratación, ventilador 


\title{
Design of an Indirect Solar Dehydrator of Fruits by Forced Convection
}

\author{
José Cabrera Escobar, \\ Raúl Cabrera Escobar, \\ Darío Guamán Lozada, \\ Diego Cajamarca Carrazco, \\ Gustavo Carrera Oña,
} Escuela Superior Politécnica de Chimborazo, Riobamba, Ecuador

\begin{abstract}
This paper serves as a guide to design a solar dehydrator by forced convection which is able to operate without power from the electricity grid, but only makes use of solar energy. This design aims to be the solution to the producers in the field. In this study, the solar dehydrator was divided into 4 fundamental parts which are: solar collector, dehydration chamber, fan, and photovoltaic panel. At the end of the research, a practical guide was obtained for the design of a solar dehydrator, taking into consideration the average global sunstroke of $4574.99 \mathrm{Wh} / \mathrm{m} 2 /$ day for Ecuador. To design a dehydrator in another country, it is important to make reference to this data. In conclusion, although it is necessary to have a dehydrator that is in excellent condition, adequate attention should also be given to the raw materials.
\end{abstract}

Keywords: Solar panel, dehydrator, dehydration chamber, fan

\section{Introduction}

En la actualidad debido a los problemas ambientales que se tiene, se trata de evitar el consumo de combustibles fósiles mismo que se utilizan para transformar materias primas en productos de valor agregado. Una de las alternativas para evitar el consumo de estos combustibles fósiles es el uso de energías alternativas, teniendo como la más importante de todas estas alternativas la energía solar (Arreola, 2015). Una fuente inagotable de energía es el sol que se encuentra aproximadamente a una temperatura de $5000{ }^{\circ} \mathrm{C}$, incluso teniendo en cuenta que solamente aprovecha el un tercio de la energía total interceptada por la atmosfera y de este tercio el $70 \%$ cae en el mar (González, 2013). La energía solar fotovoltaica ha presentado un gran 
incremento debido a la reducción de costos de todos los componentes que se tiene en un sistema solar fotovoltaico (Ospino, 2014).

La deshidratación de alimentos es un proceso de secado mediante aire caliente (Espinoza, 2016), es una de las formas más antiguas de procesar alimentos (Martínez, 2013), este proceso permite conservar los principios activos y las características organolépticas de los alimentos (Palacio, 2016), además de reducir el peso y el espacio de almacenamiento (El-Sebaii, 2012), en el proceso de deshidratación existe un intercambio de masa y de calor con el medio externo (Pirone, 2009). La presente investigación pretende diseñar un deshidratador por convección forzada debido a que de un 10 a $40 \%$ de productos se pierden por descomposición y contaminación de los productos (Guevara, 2017). El deshidratador solar consiste en calentar aire que pasa por los productos a secar, ubicados en la cámara de secado, de esta manera la humedad contenida en los alimentos se evapora (Hernández, 2015). Este deshidratador tiene la ventaja que no necesidad electricidad de la red eléctrica, su diseño fue elaborado pensando en las comunidades productoras de alimentos de nuestro país que no tiene servicio de energía eléctrica.

Este tipo de deshidratador está compuesto por un armario o cámara que puede albergar uno o dos ventiladores que hacen circular aire caliente a través de un grupo de bandejas, sobre los cuales se coloca el alimento a secar, apiladas en estantes o carretes. El aire puede ser calentado por medio de vapor, algún combustible o con corriente eléctrica, con el fin evaporar la humedad del alimento.

\section{Metodología}

La metodología que se va utilizar en esta investigación es la deductiva ya que vamos a obtener a partir de una metodología general diseño específicos de deshidratadores solares indirectos.

El deshidratador solar que se ha diseñado tiene cuatro partes fundamentales que son: colector solar, la cámara de deshidratación, ventilador y panel fotovoltaico. 


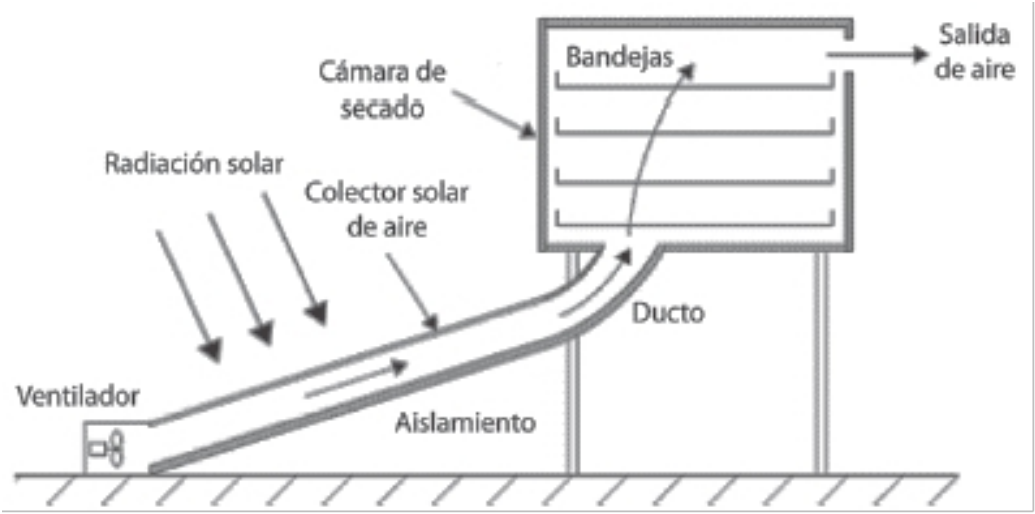

Figura 1. Elementos del deshidratador solar (García, 2012)

\section{Colector Solar}

El colector solar es el elemento del deshidratador que se encarga de calentar el aire, en la parte frontal tiene la entrada de aire, este ingreso de aire pueda darse de forma natural o de forma forzada con el ventilador. El colector solar plano es el más utilizado en pequeños productores por su bajo costo. Cuando se requiera elevar aún más la temperatura del aire es necesario aumentar la longitud del colector (Teixeira, 2016).

La ecuación 1 permite calcular el área necesaria del colector que es directamente proporcional al calor útil e inversamente proporcional a la radiación solar global y eficiencia.

Dónde:

$$
A_{c}=\frac{Q_{n}}{I n}
$$

$\mathrm{Ac}=$ Área del colector .

$\mathrm{Qu}=$ Calor útil.

$\mathrm{I}=$ Radiación solar global.

$\mathrm{n}=$ Eficiencia.

Cuando el calor no es suficiente para alcanzar la temperatura deseada, se debe puede añadir un concentrador de calor, mismo que capta la energía solar y la refleja en el colector solar (Vilcarima, 2015).

\section{Cámara de deshidratación}

Para el diseño de la cámara de secado es necesario establecer las condiciones de su estructura interna, donde serán alojadas las bandejas, la cámara de deshidratación no recibe directamente los rayos del sol, esta recibe 
el aire calentado en el colector solar. "Para el cálculo del volumen capaz de procesar de utilizar la ecuación 2.

Dónde:

$$
V_{t p}=\frac{M_{t}}{\rho_{a p}}
$$

Vtp $=$ Volumen total de producto a procesar.

$\mathrm{Mt}=$ Masa total producto a procesar.

$\rho a p=$ Densidad aparente producto a procesar.

\section{Selección de ventilador}

El ventilador es el equipo necesario para aumentar el flujo de aire de nuestro sistema, el mismo consta de tres componentes básicos que son: armazón, motor y hélices (Molina, 2017).

El objetivo de la la selección de un ventilador es, elegir aquel que satisfaga los requisitos de caudal y presión de aire, necesarios para un sistema, para la temperatura de la operación y la altitud de la instalación y además se debe determinar su tamaño, el número de revoluciones a las que debe girar el rotor, la potencia que debe ser entregada a su eje y el rendimiento. Previamente se debe determinar la masa de aire seco requerido con la ecuación 3.

Donde:

$$
m_{a s}=\frac{m_{w}}{\left(w_{4}-w_{3}\right)}
$$

Mas $=$ masa de aire seco, $\mathrm{kg}$

$\mathrm{Mw}=$ cantidad de agua a evaporar, $\mathrm{kg}$

w4 = contenido de humedad del aire a la entrada, $\mathrm{kg}$ de agua $/ \mathrm{kg}$ de aire seco w3 = contenido de humedad a la salida de la cámara de secado, $\mathrm{kg}$ agua $/ \mathrm{kg}$ aire.

Con la masa de aire se puede, determinar su volumen de aire y luego el caudal (pies cúbicos por minuto). Para la selección de ventiladores tenemos las siguientes medidas:

PCM: Pies cúbicos por minuto o metros cúbicos por hora. Este es el volumen de caudal de aire.

PE: Presión estática. Este es el término más común para identificar el empuje necesario para vencer la resistencia del sistema al flujo de aire. La unidad de medida es $\mathrm{mm} / \mathrm{cda}$ (milímetro/columna de agua) o plg/cda. 
BHP: la potencia entregada al eje del ventilador, también conocida como potencia al freno.

Con el fin de controlar la dirección y uniformidad del aire que ingresa al equipo se implementó un sistema de deflectores en el ducto de conexión entre el ventilador y el colector solar. Adicionalmente, éste se encuentra acoplado a un variador de potencia con el fin de obtener el caudal específico requerido (García, 2012).

\section{Selección del panel fotovoltaico}

La energía suministrada por el sol en forma de radiación solar se puede transformar directamente en electricidad, mediante células fotovoltaicas, aprovechando las propiedades de los materiales semiconductores.

Los componentes de un panel fotovoltaico son: Modulo Solar, Regulador de Carga, Batería, Inversor y Soportes.

Panel fotovoltaico.-Es el encargado de transformar la energía producida por los rayos de Sol a energía eléctrica de baja tensión, mediante el efecto fotoeléctrico, por lo general se trabaja con sistemas de 12 o $24 \mathrm{~V}$. Este componente se comercializa de acuerdo a la potencia requerida.

Regulador de carga. Es el encargado de regular la carga que ingresa a la batería permitiendo que la batería tenga una vida útil prolongada. Este componente es comercializado basado en su capacidad máxima de corriente a controlar (amperios).

Batería (acumulador). Este componente es indispensable en el sistema para que este funcione en días nublados o cuando no exista la suficiente energía como mover el ventilador, es el encargado de almacenar la energía eléctrica, por lo general cuando se trabaja con estos elementos se utiliza los Amperios hora (Ah).

Inversor. Este componente es el encargado de transformar la corriente continua en corriente alterna que normalmente requieren los ventiladores, si el ventilador funcionase con corriente continua (12 o $24 \mathrm{~V})$ no fuese necesario este elemento.

Para que estos componentes funcionen de manera autónoma es necesario de un controlador tal como se puede observar en la Figura 2. 


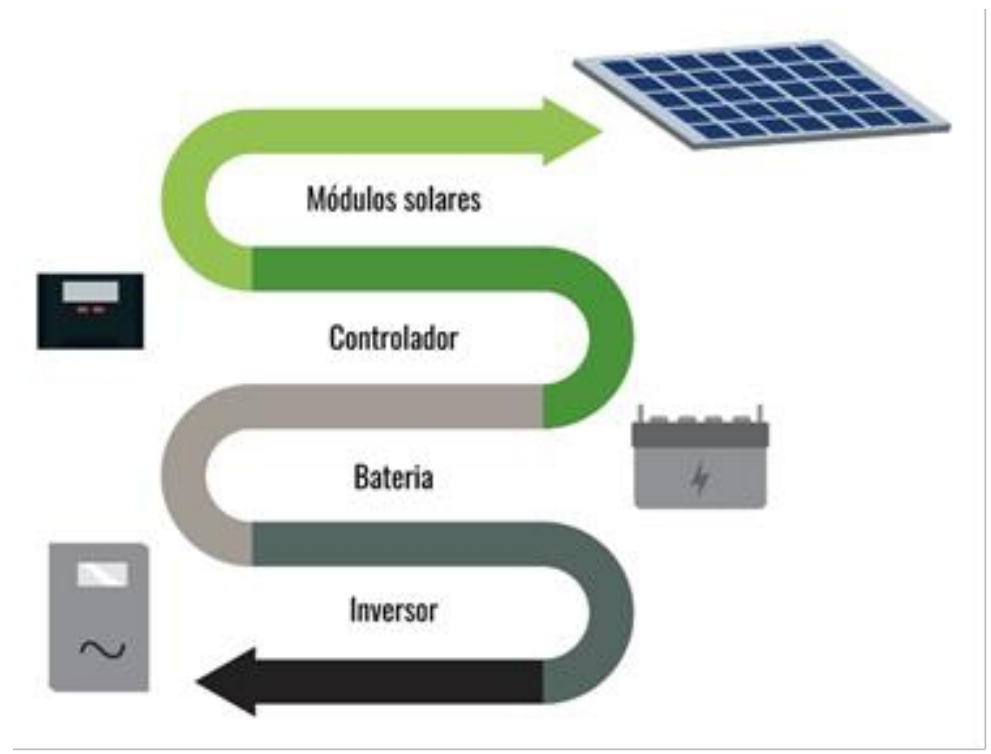

Figura 2. Esquema de un Sistema Fotovoltaico (SunSupply, 2017)

\section{Cálculos del Sistema}

Para el cálculo del calor necesario para el sistema se tiene la ecuación 4.

$$
Q=q^{*} w_{t}
$$

Donde:

$\mathrm{Q}=$ calor necesario, $\mathrm{KJ}$

$\mathrm{q}=$ consumo especifico de calor

$\mathrm{wt}=$ humedad total a extraer

Para el cálculo de la humedad a evaporar diariamente se tiene la ecuación 5.

Donde:

$$
w 1=\frac{w_{t}}{T}
$$

$\mathrm{W} 1$ = Cantidad de humedad a evaporar diariamente por $\mathrm{m} 3$ $\mathrm{T}=$ Tiempo de secado, $\mathrm{h}$.

Para el cálculo del área necesaria del secador se tiene la ecuación 6.

$$
A=\frac{Q}{Q_{i}}
$$


Donde:

$\mathrm{A}=$ Área del secador, $\mathrm{m} 2$

Qi = Energía solar aprovechada por el secador, $\mathrm{KJ}$

Para el cálculo de la energía solar aprovechada por el secador se tiene la ecuación 7.

$$
Q_{i}=H r^{*} \eta
$$

Donde:

$\mathrm{Hr}=$ Energía solar media anual recibida:

$\eta=$ Rendimiento asumido del secador: $30 \%$

Para el cálculo de la potencia total del sistema se utiliza la ecuación 8 , tomando en cuenta que 1 Wh es igual a 3600 Julios.

$$
P_{\text {total }}=P_{\text {nomin al }} * 1.15 * 1.2
$$

El factor 1.15 se utiliza por la conversión de la corriente continua (CC) en corriente alterna (CA) al pasar por el inversor, considerando una eficiencia del $85 \%$ en la conversión a plena carga del equipo.

El factor 1.2, es el factor de seguridad de sobre dimensionamiento (20\%), que tiene en cuenta el envejecimiento de los paneles y baterías, el polvo, la suciedad sobre los paneles, fallas en las conexiones eléctricas del cableado y otros accesorios eléctricos.

Para el cálculo del número de paneles o módulos necesarios se tiene la ecuación [9].

Donde:

$$
\eta=\frac{P_{\text {total }}}{W_{p}^{*} H S P}
$$

$\eta=$ Numero de paneles

Ptotal=Potencia total necesaria $(\mathrm{Wh} / \mathrm{dia})$.

$\mathrm{HSP}=$ Máxima radiación solar total que incide al día sobre los módulos, h. $\mathrm{Wp}=$ Potencia de cada panel, $\mathrm{W}$.

\section{Resultados}

Para la selección de los paneles solares que permitan el funcionamiento del ventilador y para determinar el área necesaria para el colector es de vital importancia conocer la Insolación Global promedio que para Ecuador es de 
$4574,99 \mathrm{Wh} / \mathrm{m}^{2} /$ día que se puede ver en la Figura 3 (Consejo Nacional de Electricidad, 2008). Dependiendo de la batería utilizada y los paneles fotovoltaicos será la independencia que tenga el deshidratador de la energía eléctrica. Para lograr que la materia prima tenga una buena deshidratación no solo se requiere de un deshidratador de elevada eficiencia sino también de una buena preparación de la materia prima pre-deshidratado.

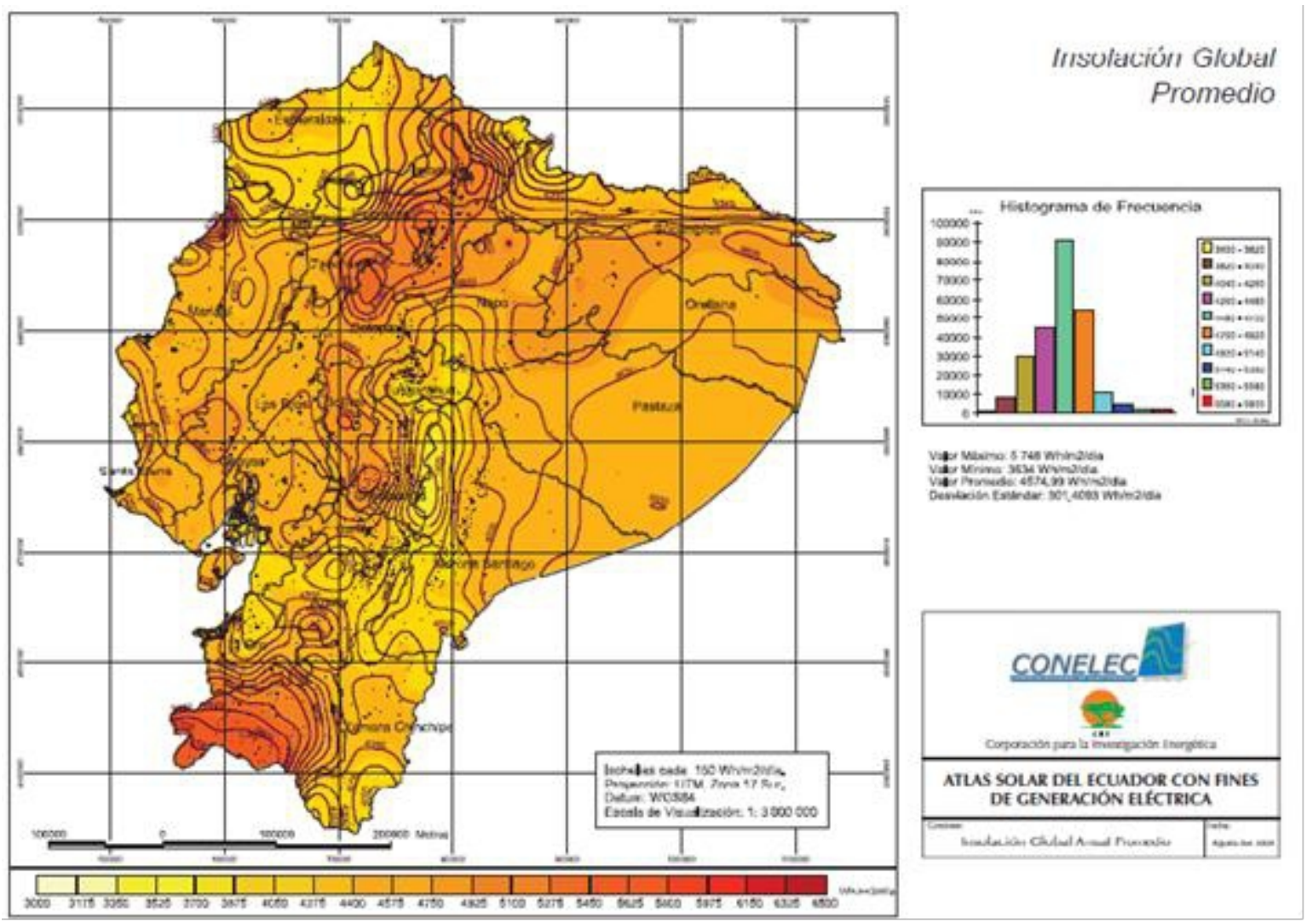

Figura 3. Insolación Global promedio del Ecuador (Consejo Nacional de Electricidad, 2008, p. 49)

\section{Discusión}

Para determinar la eficiencia térmica del sistema de deshidratación se realiza un balance de masa y energía del mismo, hallando la energía útil por la cantidad de agua evaporada y el calor latente de extracción del agua. Esta se halla con el método de peso seco de muestras horarias o diarias de producto en balanzas de precisión durante todo el proceso de secado. Simultáneamente, se halla la energía incidente o radiación solar global mediante piranómetros de alta precisión. En general, el nivel de error relativo de los instrumentos empleados no supera entre 2 y $5 \%$. (Bergues, 2014). 
Augustus León et al. (2002) analizaron distintos trabajos donde se evalúan deshidrtadores solares. Según estos autores, los parámetros que mayormente se utilizan para medir los secaderos solares pueden sintetizarse en:

- Características físicas del secadero.

- Rendimiento térmico.

- Calidad del producto desecado.

- Costos y tiempo de amortización (Iriarte, 2013).

\section{Conclusiones y recomendaciones}

Se ha desarrollado una metodología de diseño para un deshidratador solar indirecto, que va a permitir fácilmente dimensionar los componentes del sistema para su posterior construcción tomando como partida la masa de producto a deshidratadar. Para obtener mejores resultados no solo es importante el deshidratador si no también la preparación que se les da a los alimentos antes de someterle a este proceso. Con el valor obtenido de 4574,99 $\mathrm{Wh} / \mathrm{m}^{2} /$ día se puede concluir que es posible el diseño de deshidratadores solares indirectos en nuestro país porque se tiene la energía necesaria para poder deshidratar los productos.

En un próximo diseño innovador se podría plantear la necesidad de tener un almacenador de calor, para que el deshidratador tenga mayor autonomía sin necesidad de tener una resistencia eléctrica, además esto haría que se tenga un sistema más eficiente y amigable con el medio ambiente.

Se recomienda en el diseño del deshidratador incluir una toma de energía eléctrica, en caso de que ocurra algún inconveniente con el sistema de energía solar o en caso de mantenimiento para el funcionamiento del ventilador. Si se requiere aumentar aún más la temperatura al sistema se la puede hacer mediante una resistencia eléctrica.

\section{References:}

1. Arreola, R., Quevedo, A., Castro, M., Bravo, A. \& Reyes, D. (2015). Design, construction and evaluation of a solar tracking system for a photovoltaic panel. Revista Mexicana de Ciencias Agrícolas. ISSN 2007-0934.

2. Bejarano, C. \& Pérez, C. (director) (2018). Evaluación del Efecto del Uso de un Deshidratador Solar Pasivo Indirecto de Flujo Turbulento y de un Deshidratador Solar Pasivo en Propiedades Fisicoquímicas y Organolépticas de Mango, Naranja y Mandarina . Tesis de Magister en Ingeniería Agrícola. Universidad Nacional de Colombia. Bogota, Colombia. 
3. Berques, C. \& Díaz, J. (2014). Diagramas de tendencia para la generalización sostenible de secadores solares directos de productos agropecuarios. Revista Tecnología Química. ISSN 2224-6185.

4. Consejo Nacional de Electricidad (2008). Atlas solar del Ecuador con fines de generación electríca. Quito: Corporación para la Investigación Energética.

5. El-Sebaii, A. \& Shalaby, S. (2012). Solar drying of agricultural products: A review. Renewable and sustainable energy review, vol 16, pp.37-43.

6. Espinoza, J. (2016). Innovación en el deshidratado solar. Revista Chilena de Ingeniería.ISSN 0718-33

7. García, L., Mejía, M., Mejía, D. \& Valencia, A. (2012). Diseño y construcción de un deshidratador solar de frutos tropicales. Revista AVANCES Investigación en Ingeniería. ISSN 2619-6581.

8. González, C., Ponce, C., Valenzuela, R. \& Campos, D. (2013). Selección de un sistema solar fotovoltaico para un vehículo eléctrico. Revista Culcyt. ISSN 2007-0411.

9. Guevara, A. \& Sabas, J. (2017). Diseño y construcción de un deshidratador solar para fresa. Revista Jóvenes en la Ciencia. ISSN 2395-9797.

10. Gutiérrez, C., Carreño, G., Morón, A., Tobón, Y. \& López, G. (2018). Energía solar fotovoltaica en la Isla de San Andrés, Colombia Propuesta para viviendas unifamiliares. Revista Letras ConCiencia TecnoLógica.

11. Hernández, C., González, M. \& López, L. (2015). Desarrollo de un deshidratador solar indirecto por convección forzada, con colector solar de concentración usando la óptica anidólica. Memorias de la Asociación Nacional de Energía Solar. ISSN-2448-5543.

12. Iriarte, A., Bistoni, S., García, V. \& Luque, V. (2013). Evaluación de un secadero solar tendalero túnel: estudio se secado de manzanas. Revista Avances en Energías Renovables y Medio Ambiente. ISSN 2314-1433.

13. Molina, A., Castañeda, H., Salcedo, J. \& De la Cruz, L. (2017). Diseño de un banco de pruebas para ventiladores axiales en serie, norma ANSI/AMCA 210. Revista Scientia et Technica. ISSN 0122-1701.

14. Palacio, J., Bayardo, E. \& Agudelo, D. (2016). Sistema tipo marquesina de doble cámara, para el deshidratado de plantas aromáticas y medicinales empleando energía solar. Revista Politécnica. ISSN 2256-5353.

15. Pirone, B., Ochoa, M. \& Kesseler, A. (2009). Evolución de la concentración de ácido ascórbico durante el proceso de deshidratación 
de frutos de la rosa mosqueta. Revista de Investigaciones Agropecuarias. ISSN 1669-2314.

16. Ospino, A., Robles, C. \& Duran, A. (2014). Modelado y Simulación de un panel fotovoltaico empleando técnicas de inteligencia artificial. Revista Ingeniería Energética. ISSN 1815-5901.

17. SunSupply (2017). Componentes de un sistema de energía solar. Recuperado el 10 de Abril de 2019 de https://www.sunsupplyco.com/componentes-de-un-sistema-deenergia-solar/

18. Teixeira, J. \& Malpica, F. (2016). Desarrollo de un modelo matemático para dimensionar un deshidratador solar directo de cacao. Revista Ingeniería Mecánica. ISSN 1815-5944.

19. Vilcarima, C. \& Cataño, M. (director) (2015). Diseño de secador solar de piña y plátano automático. Tesis de Ingeniero Mecatrónico. Pontificia Universidad Catolica del Peru . Lima, Peru. 